\title{
Research progress on high intensity hadron accelerator in China
}

\author{
FU ShiNian \\ Institute of High Energy Physics, Chinese Academy of Sciences, Beijing 100049, China
}

Received October 5, 2012; accepted November 12, 2012

\begin{abstract}
High intensity hadron beam has wide-range important applications. In recent years, several projects with intense-beam hadron accelerator are under design, development or construction in China. We are facing a lot of challenges in beam physics and key technology of the accelerators. Beam loss and beam emittance must be minimized for such a high intensity accelerator. Space charge effect and nonlinear dynamics should be taken into account in beam physics and accelerator design. In this paper we will present the recent research progress in China on beam dynamics, new technology development and beam experiments for intense beam hadron accelerators, including linear accelerator, synchrotron and cyclotron.
\end{abstract}

hadron accelerator, intense beam, beam loss, emittance

Citation: $\quad$ Fu S N. Research progress on high intensity hadron accelerator in China. Chin Sci Bull, 2012, 57: 4663-4667, doi: 10.1007/s11434-012-5620-2

Hadron accelerator can find its important applications in a wide range. China has built a heavy ion accelerator, Heavy Ion Research Facility of Lanzhou (HIRFL) [1], with beam energy from $10 \mathrm{MeV} / \mathrm{u}$ to $1 \mathrm{GeV} / \mathrm{u}$ at Institute of Modern Physics, CAS for studies on radioactive ion beam physics, heavy ion physics, high energy density physics, super-heavy elements synthesis and atomic physics, as well as for cancer therapy. Chinese Spallation Neutron Source (CSNS) is now under construction by Institute of High Energy Physics and Institute of Physics, CAS at Dongguan, Guangdong Province. It consists of $\mathrm{H}^{-}$linear accelerator, a proton synchrotron of $1.6 \mathrm{GeV}$, a tungsten target and some spectrometers [2]. This is a platform for multi-disciplinary research and development, including materials science, biology, life sciences, chemistry, as well as physics. This will be the first pulsed neutron source in China, as a complement to the synchrotron light sources and free-electron lasers [3-6]. To meet the demands of our national long-term strategy of fission nuclear energy, Accelerator Driven Sub-critical system (ADS) was studied under the support of two phases "973 Program" for nuclear waste transmutation [7]. Based on these fundamental studies, Chinese ADS program (C-ADS) has

email: fusn@ihep.ac.cn been proposed by CAS which will use a high intensity proton linac with beam power more than $15 \mathrm{MW}$ to drive a sub-critical reactor [8]. As the first step toward such a huge accelerator and reactor system, a test facility project under CAS support has been launched in 2011 for a $50 \mathrm{MeV}$ linac construction by Institute of High Energy Physics and Institute of Modern Physics. Beijing Radioactive Ion-beam Facilities (BRIF) at China Institute of Atomic Energy is under construction with a $100 \mathrm{MeV} / 200 \mu \mathrm{A}$ cyclotron and a superconducting linear booster $[9,10]$. The high intensity proton cyclotron will be used for neutron physics, radiation physics, biology medicine studies, isotope $\mathrm{R} \& \mathrm{D}$ and so on. In addition to these big-science facilities, some small accelerators are also intensively studied for various applications, such as small neutron generator, cancer therapy, isotope production. For example, Peking University has built a $\mathrm{D}^{+}$ ion RFQ accelerator of $2 \mathrm{MeV}$ in recent years for neutron imaging and neutron activation analysis [11]. And an intense beam proton linac of $13 \mathrm{MeV}$ consisting of an RFQ cavity and a DTL tank is under construction at Tsinghua University served as a small neutron source for neutron scattering experiment and education [12].

All intense beam hadron accelerators impose a severe requirement on beam loss. This comes from the fact that the 
lost particles will eventually bombard on the accelerator components and thus make them radioactive. This prevents manual maintenance of the accelerator components which is crucially necessary for a long-term operation of the machine. For a given accelerator how much beam power we can get largely depends on how less the beam losses are. So we must take every measure to tightly control beam losses. This calls for a better understanding of the beam loss mechanism in beam physics study, a better code for the high accurate simulation of the beam transport through an accelerator, an optimized design of an accelerator with low beam loss, new technology for beam loss control and beam emittance minimization, and so on. In this paper we will present recent research progress in China on beam dynamics study, new technology development and beam experiments for hadron accelerators, including linear accelerator, synchrotron and cyclotron.

\section{Intense beam dynamics}

For the design of a high-intensity accelerator, beam dynamics issues, including space-charge effect, impedance and instability, should be addressed, so as to minimize beam loss and control emittance growth.

CSNS is designed to accelerate proton beam pulses to $1.6 \mathrm{GeV}$ kinetic energy at $25 \mathrm{~Hz}$ repetition rate, striking a solid metal target to produce spallation neutrons. The accelerator provides a beam power of $100 \mathrm{~kW}$ on the target in the first phase and then $500 \mathrm{~kW}$ in the second phase by increasing the average beam intensity 5 times while raising the linac output energy. In the phase one, an $\mathrm{H}^{-}$ion source produces a peak current of $25 \mathrm{~mA} \mathrm{H}^{-}$beam. RFQ linac bunches and accelerates it to $3 \mathrm{MeV}$. DTL linac raises the beam energy to $80 \mathrm{MeV}$. After $\mathrm{H}^{-}$beam is converted to proton beam via a stripping foil, the RCS accumulates and accelerates the proton beam to $1.6 \mathrm{GeV}$ before extracting it onto the target.

To avoid the structure instability, CSNS linac is designed with $\sigma_{x 0}, \sigma_{z 0}<90^{\circ}$, where $\sigma_{x 0}$ and $\sigma_{z 0}$ are the zero-current phase advance in transversal and longitudinal directions. To avoid the emittance growth induced by the coupling instability between transversal and longitudinal directions in a space-charge dominated beam bunch, the transversal and longitudinal beam temperature has been designed to keep equal according to the equipartitioning theory [13]:

$$
\frac{T_{\perp}}{T_{\|}}=\frac{k_{x} \varepsilon_{n x}}{k_{z} \varepsilon_{n z}}=\gamma_{0}^{2} \frac{\varepsilon_{n x}^{2}}{\varepsilon_{n z}^{2}} \cdot \frac{z_{m}^{2}}{a^{2}}=1,
$$

where $\varepsilon_{x}$ and $\varepsilon_{z}$ are the normalized RMS beam emittances in the transversal and longitudinal phase spaces; $a$ and $z_{m}$ are the radii of the bunch in the transversal and longitudinal directions; $\gamma_{0}$ is the relativistic parameter.

For the CSNS RCS, beam dynamics are summarized in
[14]. In this paper, a flexible lattice and phase-space painting at injection are designed to reduce the influence of the space-charge effect. Both correlated and anti-correlated painting schemes were carefully compared for their pro and con, and finally the anti-correlated painting scheme is selected. Impedance and instability are some other important issues in the ring design. Almost all possible impedance sources are calculated and it is found that the space-charge impedance dominates the broad-band impedance. Non-linear effects and instability usually generate not only emittance growth, but also beam halo formation. To deal with beam loss from the beam halo, a two-stage beam collimation system for transversal direction is designed in the CSNS RCS. Halo particles are firstly scattered with the primary collimator, and then the scattered particles are absorbed by the secondary collimators. Momentum collimation in longitudinal direction can be realized by a collimator located at the middle of the ring arc where a large dispersion exists. Addition of the quadrupole correctors into the RCS is also an option for beam loss control. Magnetic field errors due to non-linearity of the core material may result in tune shift, beating in bate function and dispersion function. And spacecharge effect may excite resonance of the envelope oscillation for a mismatched beam. The disturbance can be compensated by the quadrupole correctors. A detailed theoretical study and code simulations on this issue for CSNS RCS is presented in [15].

C-ADS designed a most powerful accelerator in the world with a beam power up to $15 \mathrm{MW}$, which is more than ten times higher than the present accelerator. This is very challenging and interesting task, in terms of beam dynamics design and technology development. A $10 \mathrm{~mA}, 1.5 \mathrm{GeV}$ $\mathrm{CW}$ superconducting proton linac is under studying at IHEP, CAS and planned to be built in 3 phases with energy of $50 \mathrm{MeV}, 600 \mathrm{MeV}$ and $1.5 \mathrm{GeV}$ in the following two decades, with the potential of upgradeable to $40 \mathrm{~mA}$. The linac consists of two injectors, two spoke cavity sections and two elliptical cavity sections. Different transversal focusing lattice is adopted in the linac. In the section of spoke cavity, superconducting solenoid inside the cryomodule forms a transversal focusing lattice, so as to get a short focusing period with a strong focus to the low energy beam with a strong space-charge effect. As the beam energy becomes higher in the section of elliptical cavity, room temperature quadrupole triplet is adopted between the cryomodules. The lattice is designed with the zero-current phase advance in the three directions is less than $90^{\circ}$ to avoid structural resonance in beam envelope. The equipartitioning scheme is applied in the main linac, i.e. from $10 \mathrm{MeV}$ to $1.5 \mathrm{GeV}$ [16]. Beam dynamics simulations have been performed for 10 and $40 \mathrm{~mA}$ using both codes TraceWin and Track. The calculations include 3-D space charge effects and multi-particle simulations are done using 10000 particles. Simulation for the baseline design of the main linac results in an emittance growth in $x$-plane less than $60 \%, y$-plane less than 
$50 \%$ and z-plane less than 50\%. Baseline design has no particle loss in the simulation [17].

Pursuing high beam intensity is a major developing direction for cyclotron, too. To this end, space-charge effect needs to be fully understood for a low beam loss. Previously this effect is usually described as interaction between single particles. In [18], the interaction is extended to the neighboring bunches in radial direction. In this "Start-to-Stop" model, the multi-bunch tracking is divided into three stages in sequence: star-running-stop. It imitates the stages of an accelerator's operation period in a cyclotron. In the first stage, a fresh bunch is injected without beam extraction; and then in the running stage, bunches are injected while extracted; finally in the stop stage, bunches are extracted without any fresh bunch injection. This model has implemented into a PIC code OPAL-CYCL and applied to the beam dynamics design of CYCIAE-100 compact cyclotron with a beam current $200 \mu \mathrm{A}$ at $100 \mathrm{MeV}$ output energy. The maximum design current can be up to $1 \mathrm{~mA}$ for future upgrade. Optimized design results indicate that for the initially matched beam, no space-charge induced particle losses at $1 \mathrm{~mA}$.

Simulation code for nonlinear space-charge effect study plays an essential role in both beam dynamics and accelerator design. PIC (Particle-In-Cell) model for space charge effect and Lie algebraic method for particle trajectory solution are adopted in the LEADS-DC code developed in resent for DC intense beam [19]. A self-consistent beam transport through a series of elements in third order approximation can be simulated by using this code.

\section{Key technology development}

Intense beam hadron accelerator construction demands a well preparation of a series of key technologies based on physics design.

Ion source is the starting point of a hadron accelerator beam and thus its performance has a great impact to the beam behavior in the whole downstream accelerator structures. CSNS linac has developed an $\mathrm{H}^{-}$Penning surface source in collaboration with Rutherford Appleton Laboratory, UK. The extracted $\mathrm{H}^{-}$beam reaches $53 \mathrm{~mA}$ at $50 \mathrm{keV}$ energy with a pulse length of $530 \mu \mathrm{s}$. A test run of $48 \mathrm{~h}$ was conducted with $8 \mathrm{~h}$ stable operation without any spark or other interruption [20].

LEBT transports the extracted hadron beam from ion source to the following accelerating structure, such as RadioFrequency Quadrupole (RFQ) accelerator. Beam matching is basic requirement for the LEBT for low beam loss in the downstream accelerator. In the case that the linac is used as injector to a ring, to reduce the beam loss during capture in the ring, the injected beam needs to be chopped, functioning as a pre-bunching for the ring longitudinal RF bucket. Beam should be chopped in the low energy section of a linac, such as LEBT and/or MEBT, for a low beam power dump. CSNS linac designed the LEBT with an electrostatic chopper in the space-charge neutralization LEBT [21]. It is the first try in the world. To validate the innovative design scheme, a prototype chopper was designed and manufactured. It was installed into the LEBT of the RFQ accelerator built in the first phase "973 Project" for ADS basic study. For a chopper design, fast rise/fall time is a crucial parameter because the partially deflected particles during the transit time may loss on the accelerator structure. The beam measurement at the downstream of the RFQ indicates the chopped beam pulses have a rise/fall time less than $20 \mathrm{~ns}$. This is the fastest rise/fall time in the world for LEBT chopper, owing to its special design of sloping and semi-cylindrical electrodes. In the beam experiment no perturbation to the space-charge neutralization was observed because of the full screening of the chopper located at the end of the LEBT. Owing to this achievement, CSNS linac will use this LEBT chopper only without MEBT chopper in its first phase.

For low beam loss in an RFQ accelerator, the real electric field distribution along the beam axis must be accurately coincident with the design value. Field tuning based on bead perturbation method is an important step for RFQ manufacture. Plate tuners and stick tuners are designed as static and dynamic tuners respectively for the four-rod RFQ of Peking University. A bead-pulling measurement system, consisting of a bead pulling equipment, an Agilent 8753ES network analyzer and an industrial PC, was set up [22] for the field tuning of the RFQ. After several iterations, the field flatness reached small than $5 \%$ from the original $80 \%$. It is a very effective tuning tool.

For room-temperature accelerator, Drift Tube Linac (DTL) usually follows an RFQ accelerator. CSNS linac uses an Alvarez-type DTL with four tanks to accelerate a $3 \mathrm{MeV} \mathrm{H}^{-}$ beam from the RFQ to $80 \mathrm{MeV}$. The RF frequency is $324 \mathrm{MHz}$ for high power efficiency. The high RF frequency means a very compact drift tube, which leads to many technical difficulties, especially in the low-energy section of the linac. An R\&D program for developing the key technology of the DTL was launched 5 years ago at IHEP. A $2.8 \mathrm{~m}$ long full-scale prototype has been built to validate the design principles [23]. Each drift tube houses an electromagnetical quadrupole in the FFDD lattice. Rotating coil method is applied for the magnetic center measurement. Accurate coincidence between geometrical center of the drift tube and the magnetic center of the quadrupole magnet is very essential for reducing the beam loss in the DTL. In the prototype DTL, the measurement system can reach an accuracy around $30 \mu \mathrm{m}$. At present a higher accurate measurement system has been fabricated and is under test. It is expected to reach accuracy less than $10 \mu \mathrm{m}$. In addition to magnetic field measurement, RF low-power measurements were also performed to tune the electro-magnetic properties of the cavity, including resonant frequency, field profile and stability against perturbations. A bead-pulling measurement system was set up. The tuning scheme and the procedures for ramping 
field DTL tank are established [24] and the prototype was successfully tuned to meet the tuning requirement.

RF power source for the CSNS rapid-cycling synchrotron has a fast repetition rate of $25 \mathrm{~Hz}$ and a high duty factor of $50 \%$. During the acceleration of the injected beam of $80 \mathrm{MeV}$ up to $1.6 \mathrm{GeV}$ at the extraction, the RF frequency covers a wide range from 1.02 to $2.42 \mathrm{MHz}$. Eight accelerating cavities are design to provide a total voltage of $165 \mathrm{kV}$. Ferrite rings are loaded on the cavities for tuning the RF frequency by mean of the bias current variation. A prototype cavity and a set of RF power source were developed in CSNS project for high power test. The key technology for the RF power source is related with the magic $\mathrm{T}$, the impedance transformer and the feedback amplifier. The details about design and measurement results can be found in [25].

In the compact proton cyclotron of $10 \mathrm{MeV}$ developed for PET at Huazhong University of Science and Technology, the RF power source used for driving the resonance cavity also has to track the RF frequency variation as there is no room for installation of the tuning equipment in the cavity in such a compact structure. It is realized by adjusting the output frequency of direct digital synthesizer, while measuring the ratio between forward and reflected RF power feedbacks to indentify if the generator frequency is close to the resonator frequency. And the amplitude modulation is realized by regulating the value of the external resistor. A prototype has been set up and satisfactory measurement results have been obtained [26].

Magnet is the major key component in a cyclotron. For the construction of a $14 \mathrm{MeV}$ compact cyclotron with high proton beam current up $400 \mu \mathrm{A}$ at CIAE, the main magnet has been studied in detail, for designing the magnet main parameters, searching for the method of shimming isochronous field and optimizing the magnet field in the central region [27]. The design results from 3D FEM simulations show that the magnet can meet overall requirements of the accelerator, namely CYCIAE-14. Fabrication of the magnet has started based on the design, and the shimming method has also been developed.

At CIAE, another cyclotron, named CYCIAE-10, has been built with a capability of pulsed high intensity $\mathrm{H}^{-}$ beam output at $10 \mathrm{MeV}$. To generate a pulse beam with $10 \mathrm{~ns}$ pulse length at repetition rate of $4.4 \mathrm{MHz}$ in the cyclotron, CIAE has set a test stand with a $15 \mathrm{~mA} \mathrm{H}^{-}$beam from a cusp ion source for developed the related key technology on buncher and chopper. A $70 \mathrm{MHz}$ buncher and $2.2 \mathrm{MHz}$ chopper have been constructed [28]. Beam test results show that the beam pulse structure can meet the design specification.

\section{Operation and beam experiments}

To control particle losses and to reach a high beam quality, electron cooling technology is adopted in the CSR of HIRFL. In the main ring CSRm, electron cooling helps to accumu- late more particles without particle losses, while in the experiment ring CSRe, electron cooling provides a very high beam quality for experiments, especially for the experiment with internal target. Hollow electron beam is injected into the ring and its size and density can be adjusted for optimization of the cooling effect. It is a pioneering work in the worldwide. In recent cooling experiments [29], $2.5 \times 10^{9}$ particles of ${ }^{12} \mathrm{C}^{6+}$ were accumulated in the CSRm after 10 times injection in $10 \mathrm{~s}$. Cooling accumulation and acceleration in CSRm of some other species of heavy ion is also achieved. In CSRe, $400 \mathrm{MeV} / \mathrm{u}$ and $200 \mathrm{MeV} / \mathrm{u}^{129} \mathrm{Xe}^{54+}$ were successfully cooled with internal target.

Beam commissioning of the Separated Function RFQ (SFRFQ) at Peking University has experimentally demonstrated the feasibility of this new type of RFQ accelerator. Different from normal RFQ, SFRFQ introduces gap accelerating in the quadrupole electrodes, and thus it has higher accelerating field. As a post accelerator of the existed conventional RFQ, SFRFQ can accelerate $\mathrm{O}^{+}$beam from 1.03 to $1.64 \mathrm{MeV}$ in $1.12 \mathrm{~m}$ long SFRFQ cavity, with an accelerating efficiency $60 \%$ higher than that of conventional RFQ [30]. The measured beam energy spectrum agrees well with the code simulation.

A periodical beam transport line has been set up in the downstream of the high current proton RFQ at IHEP for experimental study on beam halo formation. The beam line consists of a FODO lattice with 24 quadrupoles and a section for beam mismatch tuning with 4 quadrupoles. 14 scanners are arranged along the beam line for beam profile monitor by wires and beam halo detection by scraper. For the matched beam, we found that the measured bean profile is coincident with the simulation, but for the mismatched beam with mismatch factor $\mu=2$, there is a prominent difference between measurement and simulation [31]. More experimental study is going on for better understanding of the complex halo particle behavior in mismatched case.

We are grateful to the contributions from colleagues and friends in preparation of this article.

1 Xia J W, Zhan W L, Wei B W, et al. The heavy ion cooler-storagering project (HIRFL-CSR) at Lanzhou. Nucl Instrum Meth Phys Res, 2002, A488: 11-25

2 Fu S N, Chen H S, Chen Y, et al. Status and challenges of the China Spallation Neutron Source. In: Proceedings of IPAC2011, San Sebastián, Spain, 2011. 889-893

3 Jiang M H, Yang X, Xu H J, et al. Shanghai Synchrotron Radiation Facility. Chin Sci Bull, 2009, 54: 4171-4181

4 Feng C, Zhao Z T. Hard X-ray free-electron laser based on echo-enabled staged harmonic generation scheme. Chin Sci Bull, 2010, 55: 221-227

5 Fang W C, Gu Q, Tong D C, et al. Design optimization of a C-band traveling-wave accelerating structure for a compact X-ray Free Electron Laser facility. Chin Sci Bull, 2011, 56: 3420-3425

6 Feng C, Zhang M, Lin G Q, et al. Design study for the cascaded HGHG experiment based on the SDUV-FEL. Chin Sci Bull, 2012, 57: 3423-3429 
7 Fu S N, Fang S X, Guan X L. A high-current RFQ accelerator for the ADS system in China. J Korean Phys Soc, 2006, 48: 806-809

8 Fu S N, Chen H S, Fang S X, et al. Chinese plans for ADS and CSNS. In: Proceedings of SRF2011, Chicago, IL USA, 2011. 977-982

9 Zhang T J, Fan M W, Li Z G, et al. Research progress in high intensity cyclotron technology. Chin Sci Bull, 2009, 54: 3931-3939

10 Zhang T J, Li Z G, Chu C J, et al. Comprehensive test stand for high-intensity cyclotron development. Chin Sci Bull, 2011, 56: 238244

11 Zhang C, Guo Z Y, Schempp A, et al. Design of a high current RFQ for neutron production. Nucl Instrum Meth Phys Res, 2003, 521: 326-331

12 Wei J, Bai Y L, Cai J C, et al. The compact pulsed hadron source construction status. In: Proceedings of IPAC'10, 2010. 633-635

13 Hofmann I, Franchetti G, Boine-Frankenheim O, et al. Space charge resonances in two and three dimensional anisotropic beams. Phys Rev ST Accel Beams, 2003, 6: 024202

14 Wang S, An Y W, Fang S X, et al. An overview of design for CSNS/RCS and beam transport. Sci China Phys Mech Astron, 2011, 54(Suppl 2): 239-244

15 An Y W, Wang S. The compensation of quadrupole errors and space charge effects by using trim quadrupoles. Sci China Phys Mech Astron, 2011, 54: 214-217

16 Yan F, Li Z H, Meng C, et al. Physics design of the C-ADS main linac with two different injector schemes. In: Proceedings of HB2012, Beijing, China, 2012

17 Li Z H, Yan F, Tang J Y, et al. Beam dynamics of China ADS linac. In: Proceedings of HB2012, Beijing, China, 2012

18 Yang J, Zhang T, Lin Y, et al. High performance computation on beam dynamics problems in high intensity compact cyclotrons. Sci
China Phys Mech Astron, 2011, 54: 249-254

19 Lü J, Zhao X. LEADS-DC: A computer code for intense dc beam nonlinear transport simulation. Sci China Phys Mech Astron, 2011, 54: $236-238$

20 Wu X, Ouyang H, Chi Y, et al. Operation of the CSNS Penning surface $\mathrm{H}^{-}$ion source. Sci China Phys Mech Astron, 2011, 54: 245-248

21 Liu H, Ouyang H, Peng J, et al. A design study on the CSNS LEBT pre-chopper. Sci China Phys Mech Astron, 2011, 54: 231-235

22 Zhou Q, Zhu K, Kang M, et al. Tuner design and RF test of a fourrod RFQ. Sci China Phys Mech Astron, 2011, 54: 271-273

23 Yin X, Gong K, Peng J, et al. The R\&D status of DTL for CSNS. Sci China Phys Mech Astron, 2011, 54: 255-259

24 Peng J, Sun Z, Fu S. A new tuning procedure for the DTL RF field pattern. Chin Phys C, 2008, 32: 146-150

25 Huang W, Sun H, Zhao X, et al. A prototype RF power source for CSNS/RCS. Sci China Phys Mech Astron, 2011, 54: 218-221

$26 \mathrm{Li} \mathrm{D}, \mathrm{Hu}$ T, Liu K, et al. Design and research of RF system for $10 \mathrm{MeV}$ compact cyclotron. Sci China Phys Mech Astron, 2011, 54: 225-230

27 Zhong J, Zhang T, Li M, et al. The physics design of magnet in 14 MeV cyclotron. Sci China Phys Mech Astron, 2011, 54: 266-270

28 An S, Tin Z, Li P, et al. The design and construction of a pulsed beam generation system based on high intensity cyclotron. Sci China Phys Mech Astron, 2011, 54: 210-213

29 Yang X, Li J, Mao L, et al. Electron cooling experiments in CSR. Sci China Phys Mech Astron, 2011, 54: 274-278

30 Kang M, Lu Y, Wang Z, et al. Progress in beam commissioning of separated function RFQ accelerator. Sci China Phys Mech Astron, 2011, 54: 222-224

31 Peng J, Huang T, Liu H C, et al. The beam Halo experiment at IHEP. Chin Phys C (in press)

Open Access This article is distributed under the terms of the Creative Commons Attribution License which permits any use, distribution, and reproduction in any medium, provided the original author(s) and source are credited. 